Ann. Zootech., I978, 27 (4), 457-470.

\title{
Analyse des composantes de la productivité numérique des truies
}

\author{
c. LEGAULT \\ avec la collaboration de $C$. Finginis et Marie-Reine Pinkfitant-Langlois \\ Station de Génétique quantitative et appliquée \\ Centre national de Recherches zootechniques, I.N.R.A. \\ $7835^{\circ}$ Jouy-en-Josas (France)
}

\section{Résumé}

Les valeurs moyenties des composantes du nombre de porcelets sevrés par truie par année de vie reproductive $\left(\mathrm{P}_{n}\right)$ et par année de présence à 1'élevage $\left(\mathrm{P}_{n}^{\prime}\right)$ observées en 1977 dans 3464 troupeaux français ont été analysées en tenant compte de la taille de l'élevage. Nous soulignerons d'abord le fait que $P_{n}$, critère simple et facile à calculer, ne revêt qu'un intérêt historique en regard de $P_{n}^{\prime}$ qui est plus représentatif sur le plan économique. Quatre classes de troupeaux ont été constituées sur la base du nombre $(\mathrm{N})$ de portées sevrées au cours de la période de référence :

- $93^{\mathrm{I}}$ troupeaux pour lesquels $20<\mathrm{N}<5^{\circ}$,

- I 426 troupeaux pour lesquels $5 \mathrm{I} \leqslant \mathrm{N}<\mathrm{I} 00$,

… $7^{62}$ troupeaux pour lesquels iо $\measuredangle \mathrm{N}<180$,

- 345 troupeaux pour lesquels $N>180$.

On observe une amélioration significative de $\mathrm{P}_{n}$ et $\mathrm{P}_{n}^{\prime}$ lorsque la taille du troupeau augmente : ainsi, lorsque le nombre de portées sevrées dans l'année est inférieur à 50 ou supérieur à $\mathbf{I} 80, \mathbf{P}_{n}$ et $\mathrm{P}_{n}^{\prime}$ passent respectivement de $\mathrm{I} 8,3$ à 20,0 et de 15,9 à 17,6 porcelets sevrés par truie. Cette amélioration provient essentiellement d'une accélération du rythme de reproduction, découlant d'une réduction de 9 jours de la durée d'allaitement et de celle de l'ensemble des temps improductifs : âge à la $\mathrm{I}^{\mathrm{r}}$ mise bas arancé de $\mathrm{I} 2$ jours, intervalle sevrage-fécondation réduit de 7 jours et intervalle dernier sevrage-réforme réduit de 8 jours. En revanche, la taille de la portée est légèrement plus faible dans les plus grands élevages ( 10,0 contre 10,2$)$ alors que le taux de mortalité $y$ est réduit de I p. Ioo.

$L_{1}$ estimation des équations de régression multiple progressive a montré que $\mathrm{P}_{n}^{\prime}$ est, quelle que soit la taille du troupeau, déterminée par quatre variables significatives qui sont dans l'ordre : le nombre de porcelets nés vivants par portée, le taux de mortalité des porcelets de la naissance au sevrage, 1 'intervalle dernier sevrage-réforme et, dans trois cas sur quatre, la durée d'allaitement. L'âge à la I $^{\mathrm{re}}$ mise bas occupe la cinquième position sauf dans la seconde classe d'élevages où cette variable occupe la quatrième place. Les deux premières variables expliquent 36 à $56 \mathrm{p}$. I oo de la variance de $\mathrm{P}_{n}^{\prime}$ alors que l'ensemble des quatre variables significatives en explique $53 \mathrm{p}$. гоo dans les plus petits élevages et $7^{6} \mathrm{p}$. I oo dans les plus grands élevages.

Compte tenu de l'évolution des structures d'élevage vers des unités plus grandes et plus spécialisées, cette étude démontre que, dès maintenant, les efforts doivent porter essentiellement sur l'augmentation de la taille de la portée et la diminution du taux de mortalité des porcelets. 


\section{I. - Introduction}

Le nombre de porcelets sevrés annuellement ou " productivité numérique " des truies qui s'estime ordinairement sur la base de l'année de vie reproductive gagne, sur le plan de la signification économique, à l'être sur la base de l'année de présence dans le troupeau (LEGAULT et DAGORN, I973; LEGAULT, I978). Dans les deux cas, la productivité numérique dépend à la fois du rythme de reproduction (durée d'allaitement et intervalle sevrage-fécondation) et de la prolificité (nombre de porcelets nés vivants, nés morts et sevrés par portée). Dans la seconde situation, l'estimation de la productivité des truies s'applique à une période qui va de la fin de l'engraissement à la réforme et tient compte par conséquent des deux intervalles de temps improductifs mais coûteux qui séparent respectivement la fin de l'engraissement de la première mise bas et le dernier sevrage de la réforme.

En utilisant les informations recueillies en I977 dans les élevages français suivis par le programme national de gestion technique des troupeaux de truies (LEGAULT et al., I97I), nous avons estimé l'importance relative des composantes des deux mesures de la productivité numérique des truies en tenant compte des variations de la taille du troupeau.

\section{II. - Matériel et méthodes}

Les données recueillies dans 3464 élevages caractérisés par un nombre moyen de 97 portées sevrées dans l'année ont été considérées. Les troupeaux dont l'adhésion au programme national de gestion technique est postérieure à 1'année I976, ceux dans lesquels moins de 20 portées ont été sevrées en I977, ainsi que ceux qui présentaient des données manquantes ou aberrantes ont été éliminés. Quatre classes d'élevages ont été constituées en fonction du nombre de portées sevrées au cours de la période de référence, soit :

- 93I troupeaux avec 20 à 50 portées sevrées en I977,

- I 426 troupeaux avec 5 I à Ioo portées sevrées en I977,

- 762 troupeaux avec Ior à I80 portées sevrées en I977,

- 345 troupeaux avec plus de I80 portées sevrées en 1977 .

Cet échantillon comprenait 8 p. Ioo d'élevages de "sélection " en race pure et par conséquent une grande majorité d'élevages de "production " en race pure ou en croisement sur une partie ou la totalité de l'effectif. L'absence d'informations précises sur le type génétique de la plupart des truies en service n'a pas permis de tenir compte de ce facteur dans l'analyse statistique. Les I I variables considérées sont les moyennes annuelles d'élevage en I 977 telles qu'elles sont fournies pour la plupart aux éleveurs chaque trimestre.

- La productivité numérique ou nombre moyen de porcelets sevrés par truie par année de vie reproductive $\left(\mathrm{P}_{n}\right)$, c'est-à-dire de la première à la dernière mise bas.

- La productivité numérique par année de présence dans le troupeau c'està-dire de l'âge de 200 jours à la réforme $\left(\mathrm{P}_{n}^{\prime}\right)$ : le début de la carrière de la "truie " 
a été fixé arbitrairement à 200 jours, âge très proche de l'âge moyen à l'abattage des "porcs charcutiers" en France :

- l'âge à la première mise bas $\left(A_{1 m b}\right)$,

- le nombre moyen de porcelets nés vivants par portée $\left(\mathbf{T}_{p}\right)$,

- le nombre moyen de porcelets mort-nés par portée (M $n)$,

- le nombre moyen de porcelets morts de la naissance au sevrage (Mns),

- le taux de mortalité de la naissance au sevrage $\left(\mathrm{T}_{m}\right)$,

- la durée d'allaitement (L),

- l'intervalle sevrage-fécondation $\left(\mathrm{I}_{\mathrm{SF}}\right)$,

- 1'intervalle dernier sevrage-réforme $\left(\mathrm{I}_{\mathrm{SR}}\right)$,

- le pourcentage de $\mathrm{I}^{\mathrm{re}}$ mise bas $\left(\mathrm{P}_{1 m b}\right)$.

Les deux estimations de la productivité numérique sont associées aux autres variables par les relations :

$$
\mathrm{P}_{n}=\frac{\mathrm{T}_{p}\left(\mathrm{I}-\mathrm{T}_{m}\right) 365}{\mathrm{I}}=\frac{\mathrm{T}_{p}\left(\mathrm{I}-\mathrm{T}_{m}\right) 365}{\mathrm{G}+\mathrm{L}+\mathrm{I}_{\mathrm{sF}}}
$$

où I est l'intervalle moyen entre mises bas et $\mathrm{G}$ représente la durée de gestation supposée constante et égale à II 5 jours

$$
\mathrm{P}_{n}^{\prime}=\frac{\mathrm{N}^{\top} \mathrm{T}_{p}\left(\mathrm{I}-\mathrm{T}_{m}\right) 365}{i_{1}+\mathrm{I}(\mathrm{N}-\mathbf{I})+i_{2}}
$$

$\mathrm{N}$ représente le nombre moyen de portées sevrées par truie réformée, $i_{1}=\mathrm{A}_{1} m b-200$ est l'intervalle séparant la fin d'engraissement de la première mise bas.

$i_{2}=\mathrm{L}_{1}+\mathrm{I}_{\mathrm{SR}}$ est l'intervalle séparant la dernière mise bas de la réforme.

Après avoir estimé les paramètres statistiques et les corrélations entre variables, les équations de régression linéaire multiple progressive de $\mathrm{P}_{n}$ et $\mathrm{P}_{n}{ }_{n}$ sur les 7 variables suivantes ont été établies pour l'ensemble de l'échantillon ainsi que pour chacune des quatre classes de "taille du troupeau": $\mathrm{A}_{1 m b}, \mathrm{~T}_{p}, \mathrm{~T}_{m}, \mathrm{~L}, \mathrm{I}_{\mathrm{SF}}$, $\mathrm{I}_{\mathrm{SR}}$ et $\mathrm{P}_{1 m b}$.

L,e nombre de porcelets mort-nés $(\mathrm{M} n)$, non enregistré dans bon nombre d'élevages, ainsi que le nombre de porcelets morts en cours d'allaitement (Mns) remplacé par $T_{m}$ n'ont pas été pris en considération dans cette dernière analyse.

\section{III. - Résultats}

Les principaux paramètres statistiques (moyenne et écart-type) figurent au tableau I. La productivité numérique par année de vie reproductive est en moyenne de I 9,03 porcelets avec des valeurs extrêmes qui vont de I I à 27 porcelets. Le mode de la distribution (fig. I) est compris entre Ig et 20 porcelets. Lorsque ce paramètre est estimé sur la base de l'année de présence dans le troupeau, il se trouve réduit de 2,5 unités et relativement plus dispersé autour de la moyenne (coefficient de variation de $I_{5,4}$ contre II,8 p. IOO). Pour ce second paramètre, les valeurs s'étalent de 7 à 26 avec un mode compris entre $\mathrm{I} 6$ et $x 7$ porcelets. 


\section{TABLEAU I}

Paramètres statistiques pour chacune des quatre classes d'élevage (*) et pour l'ensemble de l'échantillon: Moyenne $(\overrightarrow{\mathrm{x}})$ et écart-type (s)

Statistical parameters of each class and for the whole sample : mean value $(\overline{\mathrm{x}})$ and standard deviation (s)

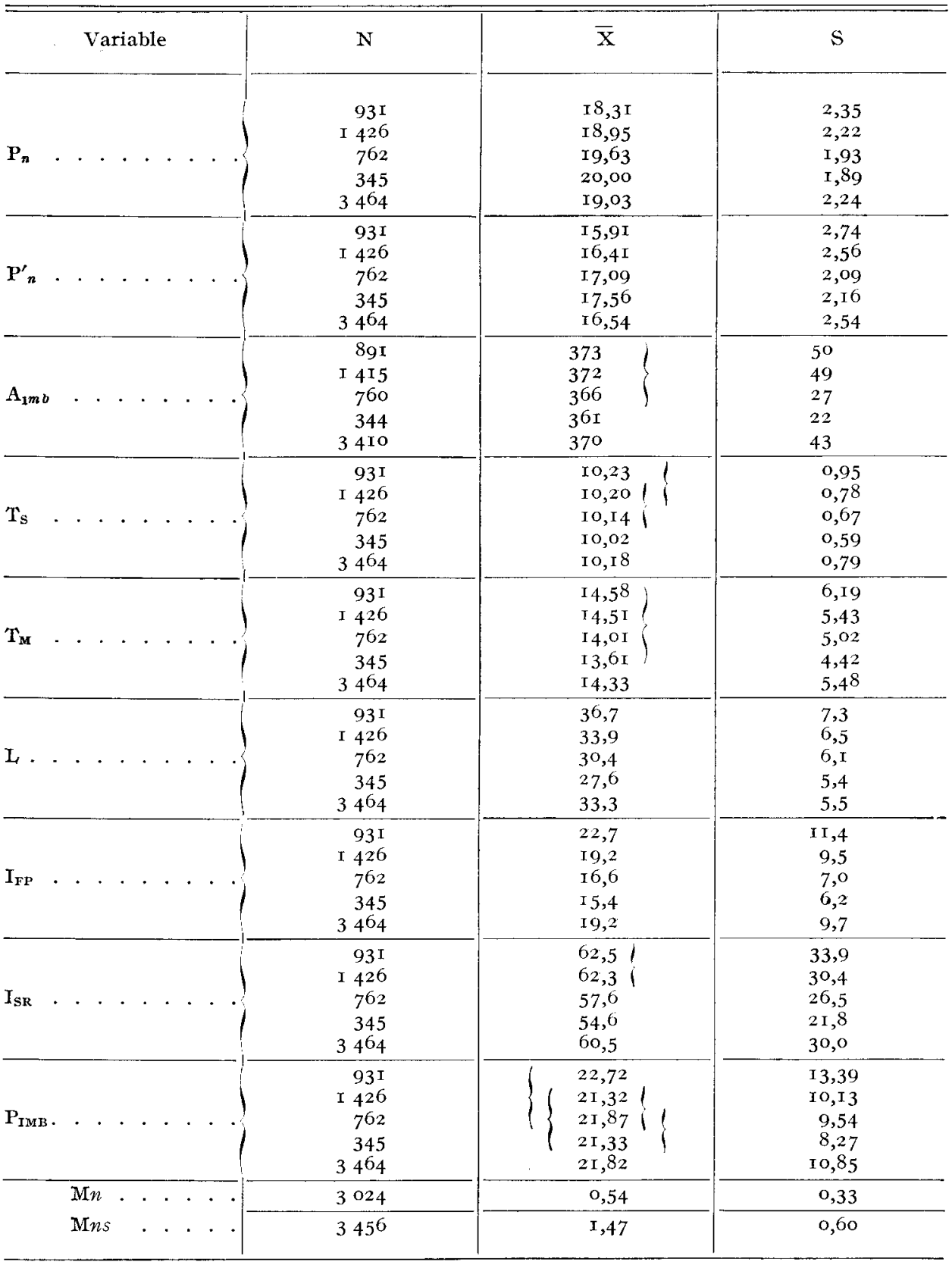

(*) Les diffétences non significatives sont réunies par une accolade. 


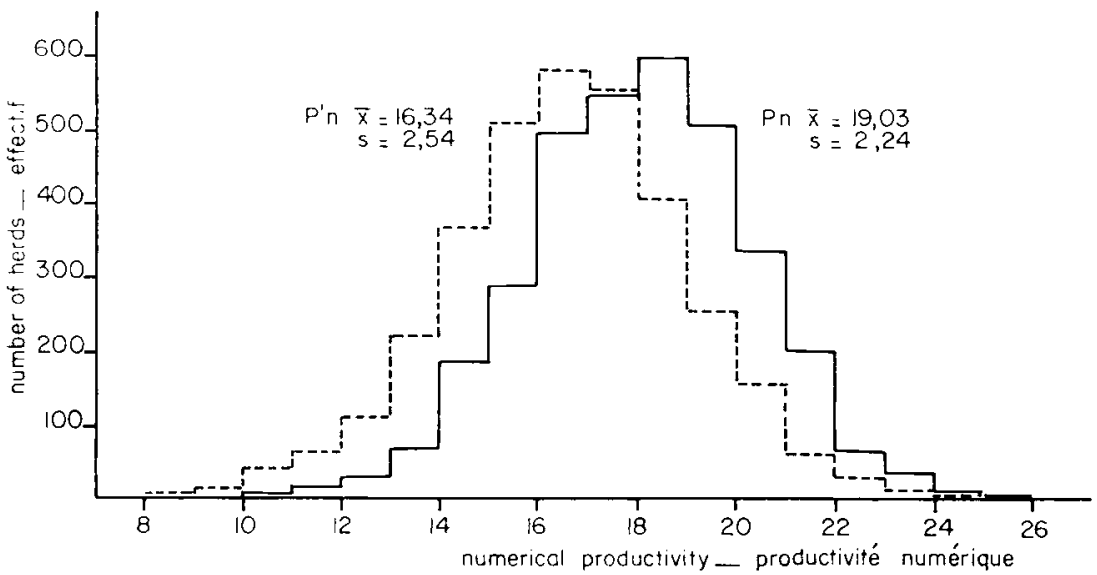

FIG. I. - Distribution des fréquences de denx estimations de la productivité numérique des truies : $\mathrm{P}_{\mathbf{n}}$ et $\mathbf{P}_{\mathbf{n}}$

Frequencies distributions of the two estimates of the mumerical productivity of sows : $\mathrm{P}_{\mathrm{n}}$ and $\mathrm{P}_{\mathrm{n}}^{\prime}$

La considération des moyennes suivant la taille du troupeau met en évidence l'augmentation générale de la productivité numérique avec la taille de l'élevage. L'effet de la classe d'effectif est hautement significatif $(\mathrm{P}<0$,or $)$ pour la plupart des variables, sauf pour la taille de la portée et la proportion de première mise bas. Les deux estimations de la productivité numérique passent respectivement de I 8,3 à 20,0 et de $I 5,9$ à I7,6 porcelets suivant que le nombre de portées sevrées dans l'année est compris entre 20 et 50 oul est supérieur à $\mathbf{I} 80$.

Cette amélioration avec la taille du troupeau provient essentiellement d'une réduction de la durée d'allaitement $(27,6$ contre 36,7 jours) et de 1'ensemble des temps improductifs : âge à la première mise bas avancé de $\mathrm{I} 2$ jours $(36 \mathrm{r}$ contre 373 jours), intervalle sevrage-fécondation réduit de 7 jours $(15,4$ contre 22,7 jours) et intervalle dernier sevrage-réforme réduit de 8 jours $(54,6$ contre 62,5 jours). Il faut noter également que dans les plus grands élevages, la taille de la portée à la naissance est légèrement plus faible (IO,O contre IO,2) mais que le taux de mortalité des porcelets y est réduit d'environ I p. Ioo (I3,6 contre I4,6 p. Ioo).

\section{IEGGNDE DU TABLEAU I. - INDEX FOR TABLE I}

$P_{n} \quad$ : Productivité numérique : Nombre de porcelets sevrés/truie/année de vie reproductive (de la $I^{\text {re }}$ à la dernière mise-bas). Numerical productivity: Number of piglets weaned per sow per year of "reproductive lite "(from the first to the last farrowing).

$\mathrm{P}_{n}^{\prime} \quad$ : Nombre de porcelets sevrés/truie/année de présence dans le troupeau (de l'âge de 200 jours à la réforme). Number of pigless weaned/sore / vear of presence on the farm (from age 2oo days up to culling).

Ans : Age à la $\mathrm{I}^{\mathrm{re}}$ mise-bas (jours). Age at first farroteing (days).

$\mathrm{T}_{p}$ : Nombre de porcelets nés vivants par portée. Number of piglets born alive per litter.

$T_{m}$ : Taux de mortalité des porcelets de la naissance au sevrage. Rate of mortality of piglets from birth to reaning.

I. : Durée d'allaitement (jours). Length of lactation (days).

ISF : Intervalle sevrage-fécondation (jours). W'eanivg-fertilization interval (days).

ISR : Intervalle dernier sevrage-réforme (jours). Last weaning-culling interval (days).

$\mathrm{P} \quad$ : Pourcentage de $\mathrm{I}^{\mathrm{re}}$ mises bas. Percentage first farrowing.

$\mathrm{M}_{n} \quad$ : Nombre de mort-nés par portée. Number of stillborn per litter.

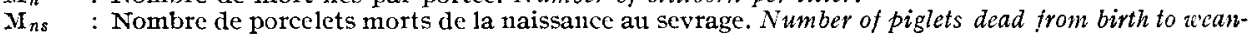
ing. 
TABLEAU 2

Corrélations linéaires entre les 9 variables

(voir légende tabl. I)

Linear correlations between the 9 variables

(see index table I)

\begin{tabular}{|c|c|c|c|c|c|c|c|c|}
\hline & $\mathrm{P}_{n}$ & $\mathrm{P}_{n}^{\prime}$ & $A_{I M B}$ & $\mathrm{~T}_{\mathbf{P}}$ & $\mathrm{T}_{\mathbf{M}}$ & L & $\mathbf{I}_{\mathbf{S P}}$ & $\mathbf{I}_{\mathrm{SR}}$ \\
\hline $\mathrm{P}_{n}^{\prime}$ & $\begin{array}{l}0,67\left(^{*}\right) \\
0,71\left(^{*}\right) \\
0,74\left(^{*}\right) \\
0,84\left(^{*}\right) \\
0,73\left(^{*}\right)\end{array}$ & & & & & & & \\
\hline $\mathbf{A}_{\text {IMB }}$ & $\begin{array}{l}-0, \mathbf{1} 2\left(^{*}\right) \\
-0,07\left(^{*}\right) \\
-0,13\left(^{*}\right) \\
-0,13\left(^{*}\right) \\
-0, \mathbf{1}\left(^{*}\right)\end{array}$ & $\begin{array}{r}-0,25\left(^{*}\right) \\
-0,28\left(^{*}\right) \\
-0,24\left(^{*}\right) \\
-0,29\left(^{*}\right) \\
-0,27\left(^{*}\right)\end{array}$ & & & & & & \\
\hline $\mathrm{T}_{\mathbf{P}}$ & $\begin{array}{l}0,60\left(^{*}\right) \\
0,55\left(^{*}\right) \\
0,51\left(^{*}\right) \\
0,61\left(^{*}\right) \\
0,52\left(^{*}\right)\end{array}$ & $\begin{array}{l}0,47\left(^{*}\right) \\
0,46\left(^{*}\right) \\
0,45\left(^{*}\right) \\
0,53\left(^{*}\right) \\
0,44\left(^{*}\right) \\
\end{array}$ & $\begin{array}{r}-0,06 \\
-0,02 \\
0,03 \\
0,02 \\
-0,02 \\
\end{array}$ & & & & & \\
\hline $\mathrm{T}_{\mathrm{M}}$ & $\begin{array}{r}\left.-0,35^{*}\right) \\
\left.-0,47^{*}\right) \\
-0,5\left(^{*}\right) \\
-0,54\left(^{*}\right) \\
-0,44\left(^{*}\right)\end{array}$ & $\begin{array}{l}-, 023\left(^{*}\right) \\
-0,33\left(^{*}\right) \\
-0,35\left(^{*}\right) \\
-0,46\left(^{*}\right) \\
-0,31\left(^{*}\right)\end{array}$ & $\begin{array}{r}0,03 \\
0,03 \\
0,06 \\
0,06 \\
-0,04\end{array}$ & $\begin{array}{l}0,29\left(^{*}\right) \\
0,23\left(^{*}\right) \\
0,23\left(^{*}\right) \\
0,11 \\
0,25\left(^{*}\right)\end{array}$ & & & & \\
\hline L & $\begin{array}{l}-0,18\left(^{*}\right) \\
-0,29\left(^{*}\right) \\
-0,3\left(^{*}\right) \\
\left.-0,35^{*}\right) \\
-0,33\left(^{*}\right)\end{array}$ & $\begin{array}{r}-0,13\left(^{*}\right) \\
-0,20\left(^{*}\right) \\
-0,19\left(^{*}\right) \\
-0,33\left(^{*}\right) \\
-0,25\left(^{*}\right)\end{array}$ & $\begin{array}{l}0,02 \\
0,04 \\
0,12\left(^{*}\right) \\
0,23\left(^{*}\right) \\
0,08\left(^{*}\right)\end{array}$ & $\begin{array}{l}0,17\left(^{*}\right) \\
0,18\left(^{*}\right) \\
0,21\left(^{*}\right) \\
0,16\left(^{*}\right) \\
0,20\left(^{*}\right) \\
\end{array}$ & $\begin{array}{l}0,02 \\
0,14\left(^{*}\right) \\
0,10(*) \\
0,27\left(^{*}\right) \\
0,11\left(^{*}\right)\end{array}$ & & & \\
\hline$I_{S F}$ & $\mid \begin{array}{r}-0,60\left(^{*}\right) \\
-0,60\left(^{*}\right) \\
-0,54\left(^{*}\right) \\
\left.-0,47^{*}\right) \\
-0,61\left(^{*}\right)\end{array}$ & {$\left[\begin{array}{r}-0,32\left(^{*}\right) \\
-0,34\left(^{*}\right) \\
-0,35\left(^{*}\right) \\
-0,33\left(^{*}\right) \\
-0,37\left(^{*}\right)\end{array}\right.$} & $\begin{array}{l}0,10\left(^{*}\right) \\
0,07\left(^{*}\right) \\
0,15\left(^{*}\right) \\
0,10 \\
0,10\left(^{*}\right)\end{array}$ & $\begin{array}{r}-0,19\left(^{*}\right) \\
-0,18\left(^{*}\right) \\
-0,12\left(^{*}\right) \\
-0,19\left(^{*}\right) \\
-0,16\left(^{*}\right)\end{array}$ & $\begin{array}{r}-0,02 \\
0,06 \\
0,05 \\
-0,05 \\
0,03\end{array}$ & $\begin{array}{c}-0,01 \\
0,02 \\
0,05 \\
-0,07 \\
0,11\left(^{*}\right)\end{array}$ & & \\
\hline $\mathrm{I}_{\mathrm{SR}}$ & $\begin{array}{r}-0, \mathrm{II}\left(^{*}\right) \\
-0, \mathrm{I} 3^{*}\left({ }^{*}\right. \\
-0,07\left(^{*}\right. \\
-0, \mathrm{I}\left(^{*}\right) \\
-0, \mathrm{I} 3\left(^{*}\right)\end{array}$ & $\begin{array}{r}-0,35\left(^{*}\right) \\
-0,39\left(^{*}\right) \\
\left.-0,42^{*}\right) \\
-0,38\left(^{*}\right) \\
-0,39\left(^{*}\right)\end{array}$ & $\begin{array}{l}0,04 \\
0,04 \\
0,04 \\
0,11 \\
0,05\end{array}$ & $\begin{array}{r}-0,06 \\
-0,06 \\
-0,08 \\
0,01 \\
-0,05\end{array}$ & $\left\{\begin{array}{c}-0,03 \\
0,01 \\
-0,09\left(^{*}\right) \\
0,05 \\
-0,02\end{array}\right.$ & $\begin{array}{l}-0,00 \\
0,07\left(^{*}\right) \\
0,02 \\
0,09 \\
0,07\left(^{*}\right)\end{array}$ & $\begin{array}{l}\left.0, \mathbf{1} 77^{*}\right) \\
\left.0, \mathbf{1} 77^{*}\right) \\
\left.0, \mathbf{I} 77^{*}\right) \\
0,24\left(^{*}\right) \\
0, \mathbf{1} 9\left(^{*}\right)\end{array}$ & \\
\hline$P_{I M B}$ & {$\left[\begin{array}{r}-0,16\left(^{*}\right) \\
-0,24\left(^{*}\right) \\
-0,22\left(^{*}\right) \\
-0,32\left(^{*}\right) \\
-0,2 \mathrm{r}\left(^{*}\right)\end{array}\right.$} & $\begin{array}{r}-0,24\left(^{*}\right) \\
-0,27\left(^{*}\right) \\
-0,27\left(^{*}\right) \\
-0,34\left(^{*}\right) \\
-0,26\left(^{*}\right)\end{array}$ & $\begin{array}{c}0,00 \\
-0,02 \\
-0,08\left(^{*}\right) \\
-0,04 \\
-0,01\end{array}$ & $\begin{array}{r}-0,26\left(^{*}\right) \\
-0,30\left(^{*}\right) \\
-0,3\left(^{*}\right) \\
-0,4^{\mathrm{I}}(*) \\
-0,30(*)\end{array}$ & $\begin{array}{c}-0,08\left(^{*}\right) \\
-0,01 \\
-0,06 \\
0,06 \\
-0,04\end{array}$ & $\begin{array}{r}-0,03 \\
-0,04 \\
-0,02 \\
-0,04 \\
-0,02\end{array}$ & $\begin{array}{l}0,05 \\
0,13\left({ }^{*}\right) \\
0,03 \\
0,08 \\
0,09\left(^{*}\right)\end{array}$ & $\begin{array}{r}-0,01 \\
0,01 \\
-0,03 \\
-0,05 \\
-0,01\end{array}$ \\
\hline
\end{tabular}

(*) Corrélation significative $(\mathrm{P}<0,05)$. Significant corrclation. 


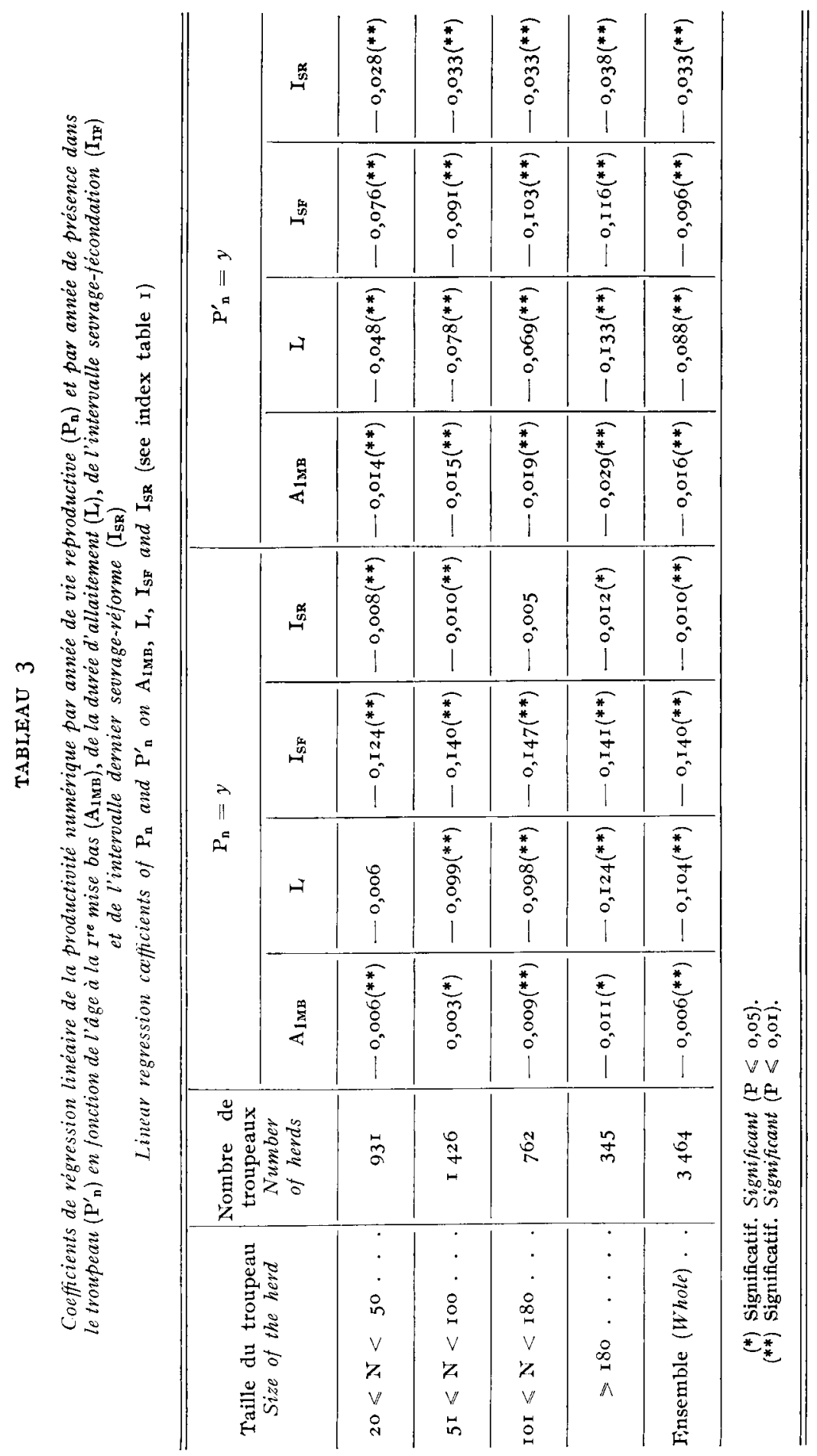


Les corrélations entre variables pour l'ensemble des troupeaux et pour chacune des quatre classes d'effectif sont rassemblées dans le tableau 2 . Les coefficients de régression linéaire des deux estimations de la productivité numérique sur l'âge à la première mise bas, la durée d'allaitement, l'intervalle sevrage-fécondation et l'intervalle dernier sevrage-réforme figurent au tableau 3. Pour une diminution de Io jours de chacune de ces variables, la productivité numérique augmente respectivement de 0,$06 ; \mathrm{I}, 04 ; \mathrm{I}, 40$ et 0 , Io porcelet par année de vie reproductive et respectivement de $0, I 6 ; 0,88 ; 0,96$ et 0,33 porcelet par année de présence dans le troupeau. Les coefficients de régression linéaire du nombre de porcelets nés vivants par portée sur la durée d'allaitement et l'intervalle sevrage-fécondation figurent au tableau 4. Pour une augmentation respective de ro jours de chacune de ces deux variables, la taille de la portée augmente de 0,22 unité et diminue de 0,13 unité.

\section{TABLEAU 4}

Coefficients de régression linéaire du nombre de porcelets nés vivants par portée $\left(\mathrm{T}_{p}\right)$ sur la durée d'allaitement ( $\left.\mathrm{I}_{4}\right)$ et l'intervalle scurage-fécondation $\left(\mathrm{I}_{\mathrm{S}} \mathrm{F}\right)$

l.inear vegression coefficients of the number of piglets born alive per litter $\left(\mathrm{T}_{p}\right)$ on the lactation lenght $(\mathrm{L})$ and on the weaning-fertilisation interval $\left(\mathrm{I}_{\mathrm{SF}}\right)$

\begin{tabular}{c}
\hline $\begin{array}{c}\text { Taille du troupeau } \\
\text { Size of the herd }\end{array}$ \\
\end{tabular}

(**) Significatif. Significant $\mathbf{P}<0$,or.

Les coefficients des équations de régression linéaire multiple progressive et leur signification statistique figurent aux tableaux 5 et 6 . Pour chacune des variables classées par ordre de détermination décroissante figure également le coefficient de détermination cumulé qui correspond au carré du coefficient de corrélation multiple cumulé. A chaque stade de l'analyse, ce chiffre représente la fraction de la variance de la variable dépendante $\left(\mathrm{P}_{n}\right.$ ou $\left.\mathrm{P}_{n}^{\prime}\right)$ expliquée par les variables indépendantes introduites.

Les variables qui déterminent significativement le nombre de porcelets sevrés par année de vie reproductive $\left(\mathbf{P}_{n}\right)$ sont pour l'ensemble de l'échantillon et les trois classes d'élevage de moindre effectif, par ordre de priorité décroissant : l'intervalle sevrage-fécondation, la taille de la portée à la naissance, le taux de mortalité des porcelets et la durée d'allaitement; les deux premières variables expliquent 52 à $60 \mathrm{p}$. roo de la variance de $\mathrm{P}_{n}$. Lans la classe d'élevages de plus grand effectif (plus de I8o portées sevrées dans l'année), c'est la taille de la portée et le taux de mortalité des porcelets qui interviennent en priorité tout en répondant de $74 \mathrm{p}$. roo de la variance de $\mathrm{P}_{n}$. 
Le nombre de porcelets sevrés par année de présence dans le troupeau $\left(\mathrm{P}_{n}^{\prime}\right)$ est également déterminé par quatre variables significatives qui sont, par ordre de priorité décroissant : la taille de la portée, le taux de mortalité des porcelets, l'intervalle dernier sevrage-réforme et, dans trois cas sur quatre, la durée d'allaitement. L'âge à la première mise bas se situe en cinquième position sauf pour les élevages de 50 à Ioo portées où il occupe la quatrième place. Les deux premières variables expliquent 36 à 56 p. Ioo de la variance de $\mathrm{P}^{\prime}{ }_{n}$ alors que l'ensemble des quatre variables significatives en explique 53 à $76 \mathrm{p}$. Ioo.

\section{IV. - Discussion et conclusions}

Dans le cadre de cette discussion, nous ne nous attarderons guère sur l'estimation de la productivité numérique par année de vie reproductive $\left(\mathrm{P}_{n}\right)$ qui, en dépit de sa simplicité, ne revêt plus qu'un intérêt historique. En revanche, nous insisterons sur le critère $\mathbf{P}_{n}^{\prime}$, estimé de l'âge de 200 jours à la réforme de la truie, qui est plus représentatif sur le plan économique. Il convient d'ailleurs de noter que $\mathrm{P}_{n}^{\prime}$ tend vers $\mathrm{P}_{n}$ lorsque le nombre moyen de portées sevrées par truie réformée $(\mathrm{N})$ augmente. Toutefois, nous devons regretter qu'il ne soit pas encore possible d'analyser un critère plus élaboré tenant compte notamment des échecs de reproduction (retards à la puberté, stérilité, accidents) chez les jeunes femelles de remplacement.

L'une des conclusions essentielles de cette étude est que l'augmentation de la taille des ateliers de production de porcelets est un facteur d'amélioration de la productivité numérique des truies. Ce résultat provient essentiellement d'une accélération du rythme de reproduction découlant de la réduction des temps improductifs : mise à la reproduction plus précoce, réduction de la durée d'allaitement et des intervalles sevrage-fécondation et dernier sevrage-réforme. Le " naisseur " spécialisé ne raisonne plus en éleveur traditionnel qui connaît ses animaux individuellement mais en gestionnaire de troupean qui élimine impitoyablement les sujets les moins productifs.

Chaque jour de retard à la première mise-bas diminue la productivité numérique des truies par année de présence dans le troupeau de o,or4 porcelet dans les plus petits troupeaux et de 0,029 porcelet dans les plus grands troupeaux. Pour un prix de revient moyen du porcelet de $25 \mathrm{~kg}$ voisin de I60 F (LEGAULT, r978), ce jour de retard représente pour l'éleveur une perte financière comprise entre 2,2 et 4,6 F, ces chiffres actualisant des résultats antérieurs obtenus d'une autre manière (LEGAUI.T et DAGORN, I973). Cependant, il faut noter que les informations sur l'âge à la première mise bas sont relativement imprécises dans les élevages de " production ». D'autre part ces résultats ne sont applicables en pratique qu'entre les âges de 340 et 400 jours : en effet, leur extrapolation à des truies plus jeunes non accompagnée d'un complément d'alimentation et de surveillance peut entraîner une chute de fertilité après la première mise bas, un retard dans le développement et par conséquent une diminution de la productivité.

La réduction d'une journée de la durée d'allaitement s'accompagne en moyenne d'une augmentation d'environ 0,1 porcelet sevré par année de présence, avec des variations comprises entre 0,05 unité dans les plus petits troupeaux et $0, I_{3}$ unité dans les plus grands troupeaux. Ces résultats confirment dans leur ensemble les estimations d'Aumaitre et al. (I975). Les résultats concernant l'influence de 
TABLEA

Coefficients de l'équation de régression linéai variables (voir

Step up linear multiple regression coefficients of

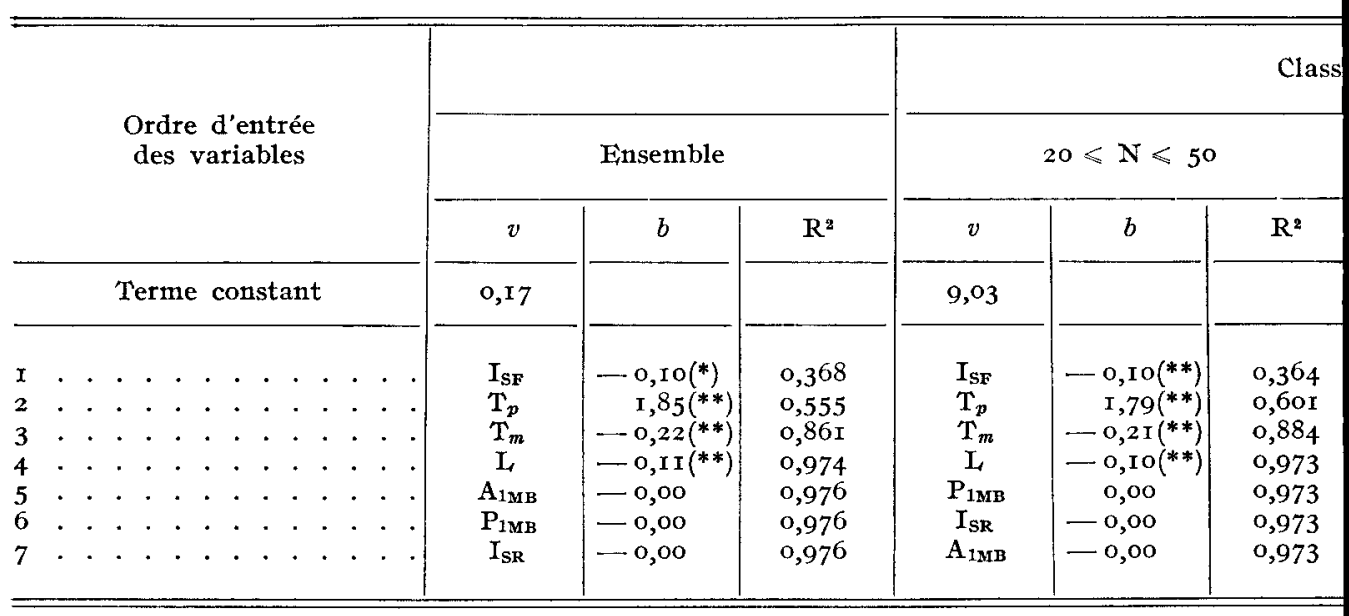

TABI.EA

Coefficients de l'équation de régression linéai variables (voir légend Step up linear multiple regression coefficients of $\mathbf{P}$

Ordre d'entrée des variables

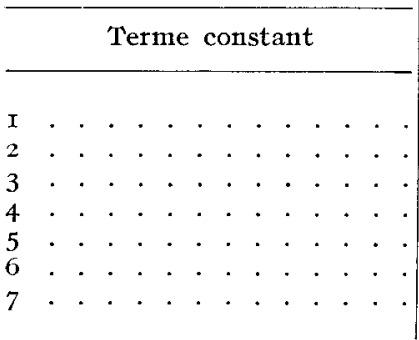

\begin{tabular}{|c|c|c|c|c|c|}
\hline \multicolumn{3}{|c|}{ Ensemble } & \multicolumn{3}{|c|}{$20 \leqslant N \leqslant 5^{\circ}$} \\
\hline$v$ & $b$ & $\mathrm{R}^{2}$ & $v$ & $b$ & $\mathrm{R}^{2}$ \\
\hline I 3.2 & & & I 2.9 & & \\
\hline $\begin{array}{c}\mathrm{T}_{3} \\
\mathrm{~T}_{m} \\
\mathrm{I}_{\mathrm{SR}} \\
\mathrm{I} \\
\mathrm{A}_{1 \mathrm{MB}} \\
\mathrm{I}_{\mathrm{SF}} \\
\mathrm{P}_{1 \mathrm{MB}}\end{array}$ & $\begin{aligned} \mathrm{r}, 6 \mathrm{r}\left({ }^{* *}\right) \\
-0, \mathrm{r} 9\left({ }^{* *}\right) \\
-0,03(* *) \\
-0,09\left(^{* *}\right) \\
-0,0 \mathrm{I} \\
-0,04 \\
-0,03\end{aligned}$ & $\begin{array}{l}0,188 \\
0,377 \\
0,518 \\
0,597 \\
0,642 \\
0,666 \\
0,680\end{array}$ & $\begin{array}{c}\mathrm{T}_{p} \\
\mathrm{~T}_{m} \\
\mathrm{I}_{\mathrm{SR}} \\
\mathrm{I}_{1} \\
\mathrm{~A}_{1 \mathrm{MB}} \\
\mathrm{I}_{\mathrm{SF}} \\
\mathrm{P}_{1 \mathrm{MB}}\end{array}$ & $\begin{aligned} & \mathrm{I}, 48\left(^{* *}\right) \\
&-0,17\left(^{* *}\right) \\
&-0,03\left(^{* *}\right) \\
&-0,08\left(^{* *}\right) \\
&-0,01 \\
&-0,03 \\
&-0,03\end{aligned}$ & $\begin{array}{l}0,208 \\
0,356 \\
0,476 \\
0,527 \\
0,564 \\
0,584 \\
0,600\end{array}$ \\
\hline
\end{tabular}

v : Variables. Variables.

$b$ : Coefficient de régression. Regression coetficient.

$\mathbf{R}^{2}$ : Carré du coefficient de corrélation multiple cumulé. Square of the cumulate multiple correlation coeffict

(**) Significatif. Significant $(\mathbf{P}<0, \mathrm{or})$. 
ultiple progressive de $\mathbf{P}_{\mathbf{n}}$ en fonction des 7 autres

gende au tabl. I)

the 7 other variables (See legende table I)

taille de troupeau

\begin{tabular}{|c|c|c|c|c|c|c|c|c|}
\hline \multicolumn{3}{|c|}{$50<N<100$} & \multicolumn{3}{|c|}{$100<N \leqslant 180$} & \multicolumn{3}{|c|}{$N>180$} \\
\hline$v$ & $b$ & $\mathrm{R}^{2}$ & $v$ & $b$ & $\mathrm{R}^{2}$ & $v$ & $b$ & $\mathrm{R}^{3}$ \\
\hline $9, \mathrm{I} 2$ & & & 8,96 & & & 7,94 & & \\
\hline $\begin{array}{c}\mathrm{I}_{\mathrm{SF}} \\
\mathrm{T}_{p} \\
\mathrm{~T}_{\mathrm{m}} \\
\mathrm{L} \\
\mathrm{A}_{\mathrm{IMB}_{\mathrm{MB}}} \\
\mathrm{P}_{\mathrm{MMB}} \\
\mathrm{I}_{\mathrm{SR}}\end{array}$ & $\begin{array}{r}-0, \mathrm{IO}\left(^{* *}\right) \\
\left.\mathrm{I}, 86^{(* *}\right) \\
-0,23\left(^{* *}\right) \\
-0, \mathrm{I}\left(^{* *}\right) \\
-0,00 \\
-0,00 \\
0,00\end{array}$ & $\begin{array}{l}0,364 \\
0,559 \\
0,880 \\
0,976 \\
0,976 \\
0,976 \\
0,976\end{array}$ & $\begin{array}{c}\mathrm{I}_{\mathrm{SF}} \\
\mathrm{T}_{p} \\
\mathrm{~T}_{m} \\
\mathrm{I} \\
\mathrm{P}_{1 \mathrm{MB}} \\
\mathrm{A}_{1 \mathrm{MB}} \\
\mathrm{I}_{\mathrm{SR}}\end{array}$ & $\begin{array}{r}-0,11\left(^{* *}\right) \\
-0,23\left(^{* *}\right) \\
1,93\left(^{* *}\right) \\
-0,12(* *) \\
-0,00 \\
-0,00 \\
0,00\end{array}$ & $\begin{array}{l}0,287 \\
0,5 \mathrm{I} 4 \\
0,849 \\
0,98 \mathrm{I} \\
0,98 \mathrm{I} \\
0,98 \mathrm{r} \\
0,98 \mathrm{I}\end{array}$ & $\begin{array}{c}\mathrm{T}_{p} \\
\mathrm{~T}_{m} \\
\mathbf{I}_{\mathbf{S F}} \\
\mathrm{L} \\
\mathrm{P}_{\mathrm{IMB}} \\
\mathbf{I}_{\mathbf{S R}} \\
\mathbf{A}_{\mathbf{M B B}}\end{array}$ & $\begin{array}{c}2,02\left(^{* *}\right) \\
-0,23\left(^{* *}\right) \\
-0,12(* *) \\
-0,12(* *) \\
-0,00 \\
0,00 \\
0,00\end{array}$ & $\begin{array}{l}0,362 \\
0,735 \\
0,881 \\
0,985 \\
0,985 \\
0,985 \\
0,985\end{array}$ \\
\hline
\end{tabular}

antiple progressive de $\mathrm{P}_{n}^{\prime}$ en fonction des 7 antres

ux tabl. I et 5)

$n$ the 7 other variables (see index tables $\mathrm{I}$ and 5 )

taille de troupeau

\begin{tabular}{|c|c|c|c|c|c|c|c|c|}
\hline \multicolumn{3}{|c|}{$5 I \leqslant N \leqslant 100$} & \multicolumn{3}{|c|}{ IOI $\quad N<I 80$} & \multicolumn{3}{|c|}{$N>180$} \\
\hline$v$ & $b$ & $\mathrm{~K}^{2}$ & $v$ & $b$ & $\mathrm{R}^{2}$ & $v$ & $b$ & $\mathrm{R}^{2}$ \\
\hline $\mathrm{I} 2.2$ & & & 14.2 & & & I 2.6 & & \\
\hline $\begin{array}{c}\mathrm{T}_{p} \\
\mathrm{~T}_{m} \\
\mathrm{I}_{\mathrm{SR}} \\
\mathrm{A}_{1 \mathrm{MB}} \\
\mathrm{L}_{4} \\
\mathrm{I}_{\mathrm{SF}} \\
\mathbf{P}_{1 \mathrm{MB}}\end{array}$ & $\begin{aligned} & \mathrm{I}, 69\left(^{(*)}\right) \\
- & 0, \mathrm{I} 9\left(^{* *}\right) \\
- & 0,03\left(^{* *}\right) \\
- & 0,01\left(^{* *}\right) \\
- & 0,08 \\
- & 0,04 \\
- & 0,03\end{aligned}$ & $\begin{array}{l}0,2 \text { I } 2 \\
0,4 \text { I I } \\
0,538 \\
0,599 \\
0,640 \\
0,659 \\
0,67 \text { I }\end{array}$ & $\begin{array}{c}\mathrm{T}_{p} \\
\mathrm{~T}_{m} \\
\mathbf{I}_{\mathrm{SR}} \\
\mathrm{L} \\
\mathrm{A}_{1 \mathrm{MB}} \\
\mathrm{I}_{\mathrm{SF}} \\
\mathrm{P}_{1 \mathrm{M} 13}\end{array}$ & $\begin{aligned} & \mathrm{I}, 58\left(^{* *}\right) \\
&-0,20\left(^{* *}\right) \\
&-0,03\left({ }^{* *}\right) \\
&-0,07\left(^{* *}\right) \\
&-0,01 \\
&-0,05 \\
&-0,03\end{aligned}$ & $\begin{array}{l}0,206 \\
0,422 \\
0,599 \\
0,660 \\
0,691 \\
0,714 \\
0,733\end{array}$ & $\begin{array}{c}\mathrm{T}_{p} \\
\mathrm{~T}_{m} \\
\mathrm{I}_{\mathrm{SR}} \\
\mathrm{L}_{1} \\
\mathrm{I}_{\mathrm{SF}} \\
\mathrm{A}_{1 \mathbf{M B}} \\
\mathrm{P}_{1 \mathrm{MB}}\end{array}$ & $\begin{array}{c}\mathrm{I}, 99\left(^{* *}\right) \\
-0,2 \mathrm{I}\left({ }^{* *}\right) \\
-0,03\left(^{* *}\right) \\
-0,10\left(^{* *}\right) \\
-0,06 \\
-0,02 \\
-0,03\end{array}$ & $\begin{array}{l}0,279 \\
0,553 \\
0,682 \\
0,756 \\
0,790 \\
0,817 \\
0,827\end{array}$ \\
\hline
\end{tabular}


la durée d'allaitement sur la taille de la portée confirment ceux d'une étude récente limitée aux races pures (LEGAUL,T, FELGINES et OWEN, I977) : chaque jour de réduction de la durée d'allaitement entraîne une diminution de l'ordre de 0,02 unité de la taille de la portée.

L'incidence favorable de la réduction d'une journée de l'intervalle sevragefécondation sur $\mathrm{P}_{n}^{\prime}$ s'accentue également avec la taille du troupeau puisqu'elle passe de 0,08 dans les plus petits à 0 , I 2 porcelet par an dans les plus grands troupeaux. Notons enfin que l'effet de la réduction de l'intervalle dernier sevrage réforme sur la productivité des truies qui est de l'ordre de 0,03 porcelet par jour est pratiquement indépendant de la taille de l'élevage.

Enfin, 1'indication d'une corrélation favorable entre la fertilité et la prolificité des truies (LEGAULT et al., I977) se trouve confirmée par la présente étude : pour une diminution d'une journée de l'intervalle sevrage-fécondation, la taille de la portée augmente en effet en moyenne de o,or3 unité.

D'une manière générale, les coefficients de régression linéaire figurant aux tableaux 3 et 4 ne peuvent être appliqués sans risque que dans des limites raisonnables au voisinage de la moyenne en raison de la non-linéarité de la plupart des liaisons.

A l'issue d'une étude portant sur les caractéristiques de $45 \mathrm{I}$ élevages suivis par le programme national de gestion technique des troupeaux de truies, TEFFENE et VANDER HAEGEN (I975) ont établi l'équation déterminant la productivité numérique par année de vie reproductive $\left(\mathrm{P}_{n}\right)$ par la méthode de la régression linéaire progressive. L'ordre d'apparition des variables était le suivant: taille de la portée à la naissance, taux de mortalité des porcelets, intervalle sevrage-fécondation et durée d'allaitement. La présente étude ne confirme ces résultats que pour la classe correspondant aux élevages de plus grande taille (plus de I80 portées sevrées par an). Pour les trois classes d'élevages de plus faible effectif, c'est en effet l'intervalle sevrage-fécondation qui intervient en premier, suivi de la taille de la portée, du taux de mortalité et de la durée d'allaitement.

Or, nous avons déjà souligné la meilleure signification économique de la productivité numérique par année de présence dans le troupeau $\left(\mathrm{P}_{n}^{\prime}\right)$. Dans cette seconde situation, quelle que soit la classe d'élevage, l'ordre d'apparition des variables est le suivant : taille de la portée, taux de mortalité des porcelets, intervalle dernier sevrage-réforme puis durée d'allaitement ou âge à la première mise bas. L'intervalle sevrage-fécondation n'intervient plus qu'en cinquième ou sixième position. La fraction de la variance de $\mathrm{P}_{n}^{\prime}$ qui est expliquée par l'ensemble des deux premières variables passe de $36 \mathrm{p}$. Ioo dans les plus petits élevages à $55 \mathrm{p}$. Ioo dans les plus grands élevages.

L'ensemble des résultats qui viennent d'être discutés attire l'attention sur la redistribution des priorités parmi les composantes de la productivité de la truie en raison de deux phénomènes : le premier est l'évolution des structures d'élevage vers des unités plus grandes, et plus spécialisées; le second phénomène qui est en partie un corollaire du précédent est 1'amélioration rapide des techniques d'élevage qui se concrétise par une réduction spectaculaire des "temps improductifs ". Comme le laissait entrevoir une étude récente (LEGAUlT et al., I977), il est dès maintenant possible d'estimer les limites (biologiques) raisonnables de cette évolution dont certaines sont déjà atteintes dans bon nombre d'élevages : 320 à 340 jours pour l'âge à la première mise-bas, 20 à 30 jours pour la durée d'allai. tement, 5 à ro jours pour l'intervalle sevrage-fécondation et $I_{5}$ à 30 jours pour l'intervalle dernier sevrage-réforme.

Il en résulte que dès à présent ainsi qu'au cours des années qui viennent, les 
composantes essentielles de la productivité des truies sont la taille de la portée à la naissance et le taux de survie des porcelets jusqu'au sevrage.

Nous ne développerons pas ici les différents chapitres d'une récente mise au point sur les possibilités d'amélioration génétique de la productivité des truies (LEGAUi,T, I978). Remarquons d'abord que la taille de la portée a été plutôt délaissée par les sélectionneurs au cours de la dernière décennie en raison de la lenteur du progrès attendu en regard de celui espéré d'un recours aux croisements. Pour un taux de sélection de $5^{\circ} \mathrm{p}$. I oo appliqué aux truies classées en fonction d'un indice de prolificité intra-troupeau, le progrès génétique théorique est de 1'ordre de 0,5 porcelet par portée tous les dix ans (L,EGAUL'T, I970; LEGAUI, $T$ et al., I97I). L'usage des croisements est source d'un progrès plus spectaculaire atteint en deux générations qui est de l'ordre de $8 \mathrm{p}$. Ioo sur la taille de la portée à la naissance et de i2 p. Ioo au sevrage (SElliter, I97o; SEldier, I976; Olifivier et al., I978). Sélection et croisements apparaissent donc comme deux techniques complémentaires. D'autres méthodes d'amélioration génétique de la prolificité telles que l'usage en insémination artificielle de verrats appartenant à une lignée " hyperprolifique " (LEGAULT et GRUAND, I976) ou l'utilisation en croisement de certaines races asiatiques hautement prolifiques peuvent également être envisagées à l'avenir.

Le taux de mortinatalité qui est voisin de $5 \mathrm{p}$. Ioo figure pour l'instant parmi les constantes biologiques de l'espèce. Par contre, le taux de mortalité de la naissance au sevrage, qui a diminué sensiblement au cours des dernières années (LEGAULT et al., I977), est réduit dans les plus grands élevages (I3,6 contre I4,6 p. Ioo) et semble encore très largement améliorable; on note en effet que ce taux est in férieur à ro p. roo, parfois à 5 p. roo, dans de nombreux élevages français. Si la pratique des croisements est susceptible de diminuer cette mortalité de 6 à 8 p. roo, ce sont avant tout les installations de mise bas, la surveillance des éleveurs et l'état sanitaire qui jouent un rôle déterminant.

En définitive, il semble que, dans le proche avenir, l'amélioration de la productivité numérique des truies soit avant tout du domaine de la génétique et de la pathologie.

Accepté pour publication en aồt 1978 .

\section{Remerciements}

L'auteur tient à remercier MM. A. Aumatref, J. Dagokn et P. Skiflikr pour l'aide et les suggestions apportées lors de l'interprétation des résultats et de la rédaction de ce texte.

\section{Summary}

Analysis of the components of sow's numerical productivity

The average values of the components of the number of piglets reared per sow per year of reproductive life $\left(\mathrm{P}_{n}\right)$ and per year of presence on the farm $\left(\mathrm{P}_{n}^{\prime}\right)$ observed in 1977 in 3464 French pig herds were analyzed taking into account the size of the herd. We should like first of all to point out the fact that although $P_{n}$ is an easy criterion to derive, it is of minor importance 
compared to $\mathrm{P}_{n}^{\prime}$ which is closer to the economic reality. Four classes of herds were set up on the basis of the number (N) of litters weaned in the course of the reference period:

-. 934 herds with

- I 426 herds with

- 762 herds with

- 345 herds with

$$
\begin{array}{r}
20 \leqslant N \leqslant 50, \\
5 I \leqslant N \leqslant I O O, \\
I O I \leqslant N \leqslant I 80, \\
N>I 80 .
\end{array}
$$

A significant improvement in $P_{n}$ and $P_{n}^{\prime}$ is observed as the herd size increases: thus, as the number of litter weaned during the year is less than 50 or greater than $\mathrm{I} 8 \mathrm{o}, \mathrm{P}_{\mathrm{n}}$ and $\mathrm{P}_{n}^{\prime}$ go respectively from 18.3 to 20.0 and from I 5.9 to I 7.6 piglets weaned per sow. This improvement is due essentially to an acceleration in the rhythm of reproduction as a result of a reduction by 9 days of the milking period, by 12 days of age at first farrowing, by 7 days of the weaning-fertilization period and by 8 days of the last weaning-culling period. On the other hand, litter size is slightly smaller in the biggest herds (I0.O as against 10.2) whereas the mortality rate is I p. IOo lower in these herds.

Step up multiple linear regression equations show that whatever the herd size, $\mathrm{P}_{n}^{\prime}$ is determined by 4 significant variables in the following order: litter size at birth, mortality rate from birth to weaning, the last weaning-culling interval and, in 3 out of 4 cases, the length of the milking period. The age at first farrowing is in fifth place except in the second class where it is in fourth place. The first two variables account for from $3^{6}$ to $56 \mathrm{p}$. I oo of the variance of $P_{n}^{\prime}$, whereas the four significant variables taken together account for $53 \mathrm{p}$. roo of the variance in the smallest herds and $76 \mathrm{p}$. I oo in the largest.

Taking into account the tendency for herds to become larger and more specialized, this study shows that, from now on, efforts should be directed at increasing litter size at birth and at lowering mortality rate among suckling piglets.

\section{Références bibliographiques}

Aumaitre A., Perez J. M., Chauvel J., 1975. Effet de 1'habitat et de l'âge au sevrage sur les composantes de la productivité des truies en France. J. Rech. Porcine en France. I.N.R.A.I.T.P., Ed., Paris, LIII-LXVI.

I.EGAUr'T C., 1970. Recherche d'un taux optimum de sélection des jeunes truies sur la prolificité de leur mère. J. Rech. Porcine en France. I.N.R.A.-I.T.P., Ed., Paris. 24 I-249.

LEGaUlT C., I978. Génétique et reproduction chez le porc. J. Rech. Porcine en France. I.N.R.A.-I.T.P., Ed., Paris, 43-6o.

IEGAULT C., DAGORN J., I973. Incidence de 1'âge à la première mise bas sur la productivité de la truie. J. Rech. Porcine en France. I.N.R.A.-I.T.P., Ed., Paris, 227-237.

Legaulí C., Gruand J., I976. Amélioration de la prolificité des truies par la création d'une lignée "hyperprolifique " et I'usage de 1 insémination artificielle : principe et résultats expérimentaux préliminaires. J. Rech. Porcine en France. I.N.R.A.-I.T.P., Ed., Paris, $201-206$.

Legault C., Felgines C., OWEN J., I977. Analyse statistique des composantes de la productivité numérique des truies de quatre races françaises. J. Rech. Porcine en France. T.N.R.A.I.T.P., Ed., Paris, 57-62.

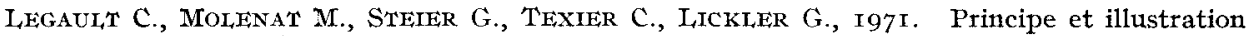
d'un programme d'interprétation mécanographique des performances d'élevage des truies. J. Rech. Porcine en France. I.N.R.A.-I.T.P., Ed., Paris, II-I4.

Olitvier I., Legault C., Molenat M., Sellier P., I978. Les recherches en Génétique porcine et leurs applications: Un bilan de la période 1969-1977. J. Rech. Porcine en France. I.N.R.A.I.T.P., Ed., Paris, 27-42.

SELIIER P., I970. Hétérosis et croisement chez le Porc. Ann. Génét. Sél. anim., 2, 145-207. SELLIER P., I976. The basis of crossbreeding in pigs: a review. Livest. Prod. Sci., 3, $203-226$. TEFFene: O., VanderhaEgen J., I975. Facteurs de productivité des élevages de truies. $J$. Rech. Porcine en France. I.N.R.A.-I.T.P., Ed., Paris, XXXI-XI.II. 